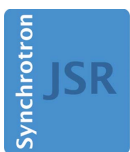

JOURNAL OF SYNCHROTRON RADIATION
Keywords: small-wedge synchrotron crystallography (SWSX); protein crystallography; radiation damage; de novo phasing; dose

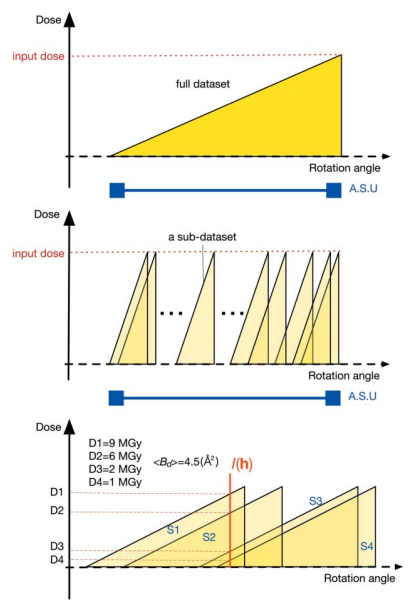

OPEN Ð ACCESS

Published under a CC BY 4.0 licence

\section{Guidelines for de novo phasing using multiple small-wedge data collection. Corrigendum}

\author{
Seiki Baba, ${ }^{a}$ Hiroaki Matsuura, ${ }^{b}$ Takashi Kawamura, ${ }^{\text {a Naoki Sakai, }}$ \\ Yuki Nakamura, ${ }^{\text {a }}$ Yoshiaki Kawano, ${ }^{b}$ Nobuhiro Mizuno, ${ }^{\text {a }}$ Takashi Kumasaka, \\ Masaki Yamamotob and Kunio Hirata ${ }^{b}$ *
}

aProtein Crystal Analysis Division, Japan Synchrotron Radiation Research Institute, 1-1-1 Kouto, Sayo, Hyogo 679-5198, Japan, and ${ }^{\mathbf{b}}$ Life Science Research Infrastructure Group, RIKEN SPring-8 Center, 1-1-1 Kouto, Sayo-Cho, Sayo-gun, Hyogo 679-5148, Japan. *Correspondence e-mail: kunio.hirata@riken.jp

A figure in the article by Baba et al. [(2021), J. Synchrotron Rad. 28, 1284-1295] is corrected.

The $x$-axis ranges (0-120) shown in Fig. 2 on p. 1290 of the article by Baba et al. (2021) are incorrect. The correct figure with the $x$-axes in the range $0-200$ is published here.
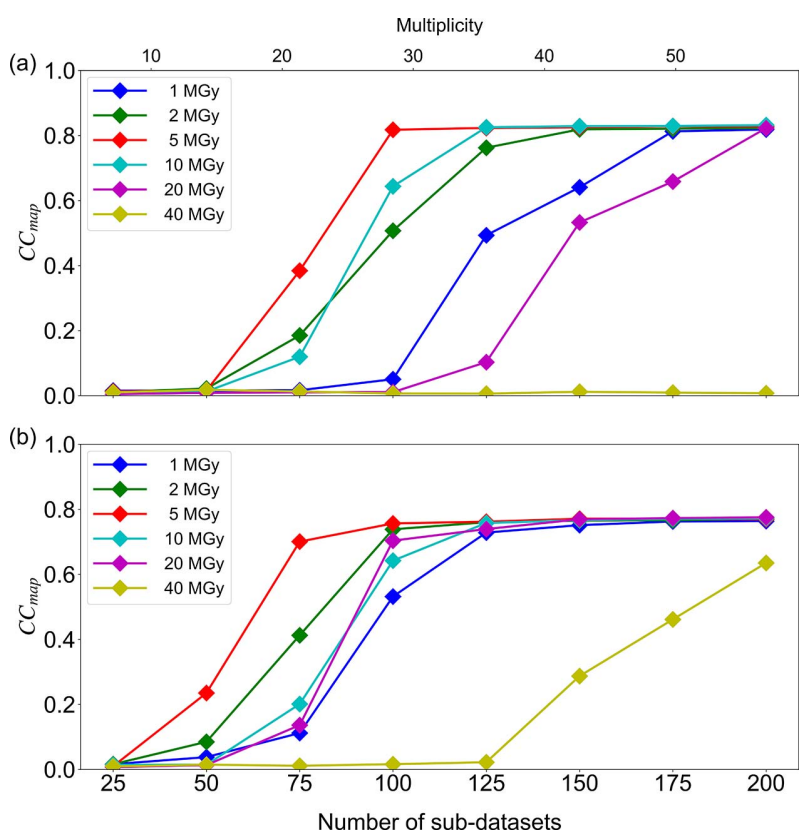

Figure 2

Correlation between the number of data merged for each dose and $\mathrm{CC}_{\text {map }}$ for $(a) \lambda=1.4 \AA$, (b) $\lambda=1.7 \AA$. Mean values of the correlation coefficient $\left(\mathrm{CC}_{\text {map }}\right)$ derived from the phase determinations for ten randomly selected merged sub-datasets were plotted against the number of sub-datasets.

\section{References}

Baba, S., Matsuura, H., Kawamura, T., Sakai, N., Nakamura, Y., Kawano, Y., Mizuno, N., Kumasaka, T., Yamamoto, M. \& Hirata, K. (2021). J. Synchrotron Rad. 28, 1284-1295. 